$5-1-1976$

\title{
Multiple Rainbows from Single Drops of Water and Other Liquids
}

Jearl D. Walker

Cleveland State University

Follow this and additional works at: https://engagedscholarship.csuohio.edu/sciphysics_facpub

Part of the Physics Commons

How does access to this work benefit you? Let us know!

\section{Publisher's Statement}

Copyright 1976 American Association of Physics Teachers. This article may be downloaded for personal use only. Any other use requires prior permission of the author and the American Association of Physics Teachers. The following article appeared in American Journal of Physics 44 (1976): 421-433 and may be found at http://ajp.aapt.org/resource/1/ajpias/v44/i5/ p421_s1?isAuthorized=no.

\section{Original Citation}

Walker, Jearl D. "Multiple Rainbows from Single Drops of Water and Other Liquids." American Journal of Physics 44 (1976): 421-433.

\section{Repository Citation}

Walker, Jearl D., "Multiple Rainbows from Single Drops of Water and Other Liquids" (1976). Physics Faculty Publications. 80.

https://engagedscholarship.csuohio.edu/sciphysics_facpub/80

This Article is brought to you for free and open access by the Physics Department at EngagedScholarship@CSU. It has been accepted for inclusion in Physics Faculty Publications by an authorized administrator of EngagedScholarship@CSU. For more information, please contact library.es@csuohio.edu. 


\begin{abstract}
Jearl D. Walker
Physics Department, Cleveland State University, Cleveland, Ohio 44115

(Received 1 May 1975; revised 20 August 1975)

The scattering of light by spherical drops and the theory of rainbows are reviewed in detail to predict the angular positions of rainbows from single drops. The angular positions of the first 13 rainbows of water, observed from a drop suspended in a student spectrometer, were compared to these theoretical positions. Since the rainbows were dependent on the refractive index, the positions shifted with drops of other fluids having other refractive indices. Light corn syrup yielded 11 rainbows, including the 17 th-order one. The light emerging from the drops was white except for the multicolors of the rainbows and the faint blue of the rays that had been nearly tangentially incident to the drops. With a hand-held polarized filter, all of the observed rainbows appeared to be polarized parallel to the plane of incidence. Using the $6328-\AA$ light from a student He-Ne laser, one of the interference patterns composing all natural rainbows was created and photographed. The angular positions of the principal maxima of this pattern differed from the theoretical rainbow positions predicted by geometrical optics by amounts dependent on the drop radius.
\end{abstract}

Somewhere over the rainbow, way up high, There's a land that I heard of once in a lullaby. Somewhere over the rainbow skies are blue, And the dreams that you dare to dream really do come true. ${ }^{1}$

\section{INTRODUCTION}

Rainbows are the most beautiful sight in the sky. Scientists and laymen alike have studied them for centuries and have honored them innumerable times in song and verse. Exposure to such splendor not only introduces a student to geometrical optics, dispersion, and wave interference, but will also imbed a permanent canvas of colors in the student's memory of physics. Once rainbows are understood, each fresh and unexpected observation of them will stimulate the memory and reinforce the physics.

A single reflection of sunlight inside falling raindrops produces the primary rainbow, while a double reflection yields the secondary rainbow. ${ }^{2-12}$ Some rays could contribute to other rainbows after experiencing additional internal reflections. These other rainbows are never seen in nature, however, since they are weaker than the background sky brightness, the light reflected from the outside surface of the raindrops, or the light transmitted through the raindrops with no internal reflections.

There have been no verified observations of any but the first two rainbows in a natural landscape. Yet in 1868 Billet $^{13}$ was able to detect the first 19 rainbows from a thin stream of water which was illuminated with various colored lamps. Using a spectrometer to examine drops of water and other liquids, a student can see not only many rainbows but also other color and polarization details predicted by geometrical optics for the scattering of light from drops.

Theoretical rainbows will be reviewed in two ways in this paper. First, the scattering angles, widths, and color sequences of the first 20 rainbows will be determined with geometrical ray tracing. The theoretical intensities of these rainbows, as well as the intensity of light scattered by drops at other angles, will also be calculated. Then, second, after the relative phase of the rays is included in the calculations, the rainbow will be shown to be an interference pattern with several features inexplicable with the strictly ray tracing analysis. (a)

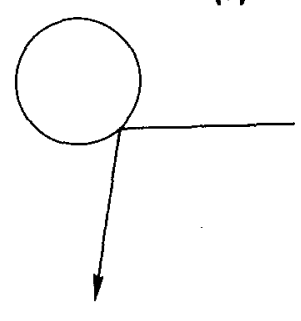

(b)

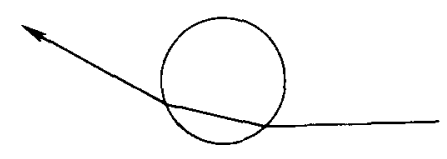

(c)

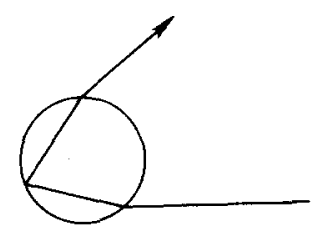

Fig. 1. Light can be reflected from the outer surface (a), transmitted across the drop with no reflections inside the drop (b), or transmitted with one or more internal reflections (c).

\section{MULTIPLE RAINBOWS}

The study of rainbows is the study of the deflection of light rays by a spherical water drop. When a raindrop is illuminated with sunlight, some light is reflected by the drop's outer surface [Fig. 1(a)], while some is refracted into the drop, crosses the drop, and is refracted out of the drop on the other side [Fig. 1(b)]. Still another fraction of the light which is refracted into the drop eventually emerges by a second refraction, but suffers one or more reflections inside the drop [Fig. 1(c)]. We shall first consider those rays reflected only once inside the drop since they are the source of the primary rainbow.

The first basic explanation for the primary rainbow, attributed to Descartes, ${ }^{5,14}$ places the rainbow at the angle of the densest clustering of the rays leaving the drop after those rays are reflected once inside the drop. In Fig. 2 the single reflection of several rays at various incident angles to the drop's surface is illustrated. The density of emerging rays is greatest around ray $f$ which has been deviated the least by the drop from the initial direction of the rays. Therefore, finding the angle of the rainbow with respect to the initial direction of the rays amounts to finding the smallest angle of deviation suffered by the rays. 


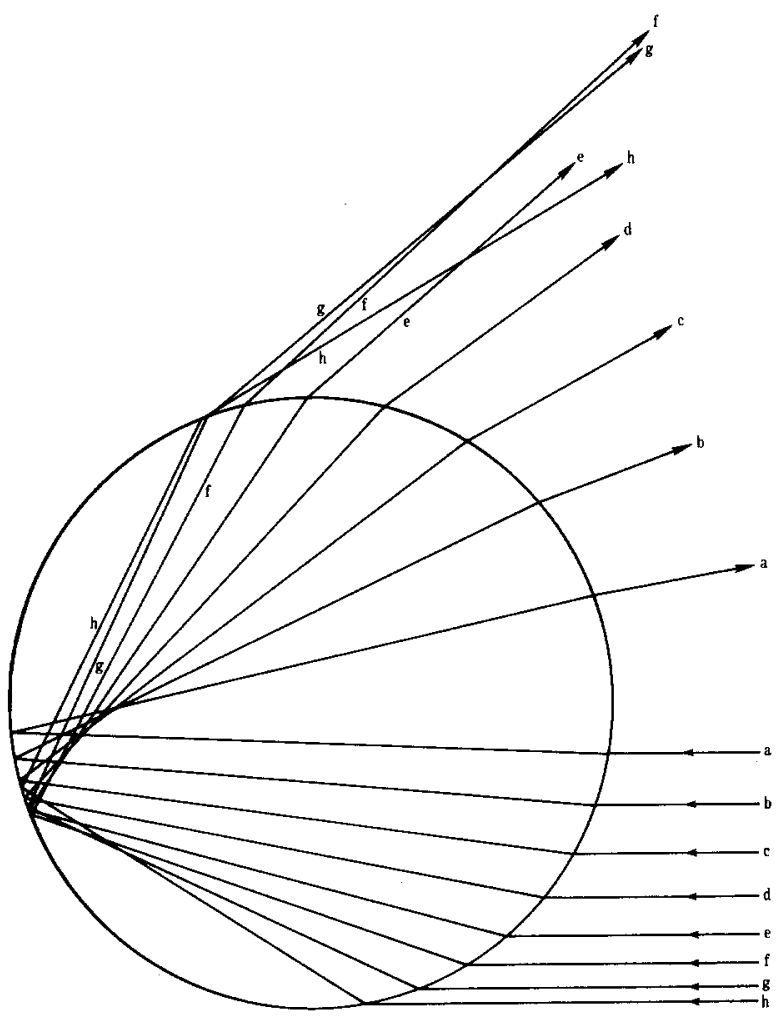

Fig. 2. Some of the rays experiencing a single reflection inside a water drop are shown. The ray experiencing the least deviation, ray $f$, is the Cartesian ray of the primary rainbow. A fully illuminated drop has a distribution of rays symmetric about the ray of normal incidence.

Upon entering a drop, a ray is deviated by an angle equal to $i-r$, where $i$ and $r$ are the angles of incidence and refraction (Fig. 3), which are related by Snell's law. An internal reflection causes a further deviation by an angle equal to $180^{\circ}-2 r$. Upon leaving the drop, there is a final deviation by an angle equal to $i-r$. Thus, the total deviation of a ray reflected once inside the drop is

$$
\theta=180^{\circ}+2 i-4 r .
$$

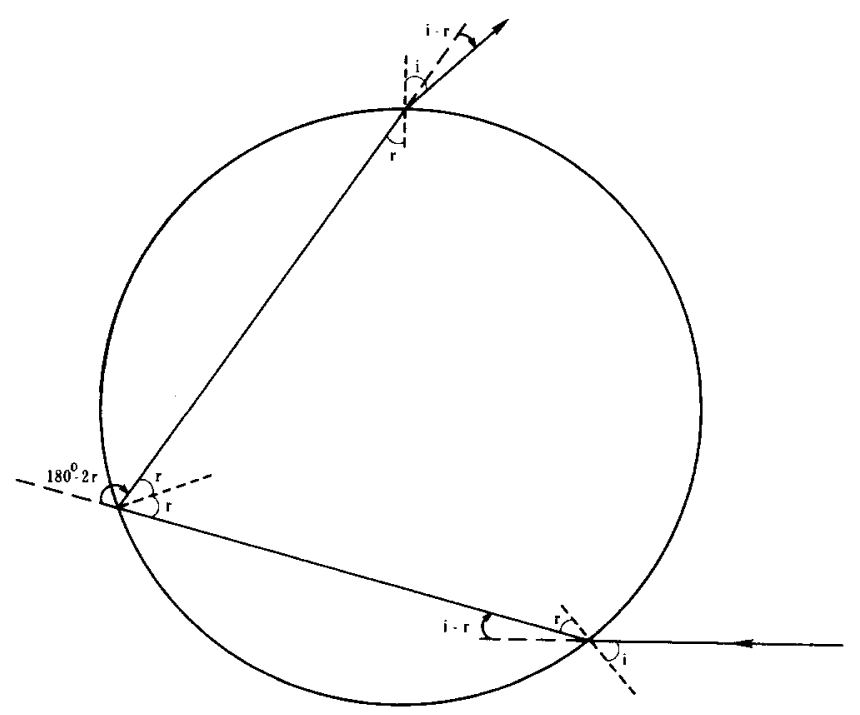

Fig. 3. Angles indicated are those needed to calculate the angle of deviation between the initial and final directions of a ray suffering one internal reflection.

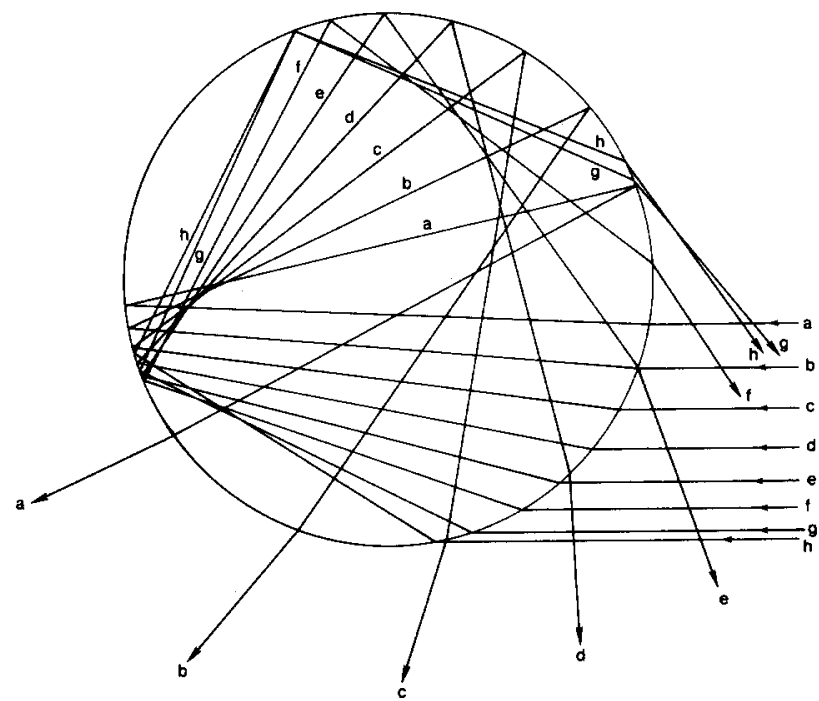

Fig. 4. Some of the rays undergoing two internal reflections are given. The ray experiencing the least deviation, ray $g$, is the Cartesian ray of the secondary rainbow.

The least deviation possible for a ray undergoing one internal reflection can be found by setting $d \theta / d i=0$ to find the extremum of (1). Doing this, and using Snell's law to eliminate the angle $r$, we find the $i$ for the case of least deviation as

$$
\cos i=\left[\frac{1}{3}\left(n^{2}-1\right)\right]^{1 / 2}
$$

where $n$ is the index of refraction. The angle $\theta$ of least deviation is obtained by solving for $i$, using Snell's law to find the corresponding $r$, and finally substituting the $i$ and $r$ into (1). Since the Cartesian rainbow appears at the angle of least deviation, we shall denote this angle by $\theta_{c}$. Similarly, the ray entering the drop and then exiting at $\theta_{c}$ will be termed the Cartesian ray. In Fig. 2 the Cartesian ray is ray $f$, which is incident at an angle of $59^{\circ}$ and exits after a deviation of about $138^{\circ}$. The other rays of Fig. 2, all of which experience a greater deviation than ray $f$, are incident at intervals of $10^{\circ}$.

Although Descartes' calculations of $\theta_{c}$ were correct, his explanation of why there are colors at that angle was erroneous. ${ }^{15}$ It was not until Newton's announcement of the dispersion of light by water that there was a correct explanation of the colors. ${ }^{16}$ Simply stated, a different index of refraction is associated with each wavelength of light and thus, by the calculations above, each wavelength will have a slightly different $\theta_{c}$. The $n$ for red light in water is about 1.331 and for blue light is about 1.343. The corresponding $\theta_{c}$ is $137.63^{\circ}$ for red light and $139.35^{\circ}$ for blue light. The intermediate colors have intermediate angles for the brightest light. The rainbow, therefore, has an angular width ${ }^{17}$ of approximately $1.72^{\circ}$.

Similar considerations of minimum deviation can be made for more internal reflections. The greatest clustering of rays for two internal reflections is, again, at the angle of least deviation as indicated by Fig. 4. The Cartesian ray $g$ is incident at an angle of about $72^{\circ}$ and exits after a deviation of about $232^{\circ}$. The other rays of Fig. 4, which are incident at intervals of $10^{\circ}$, all experience greater deviations. Hence, the secondary rainbow corresponds to ray $g$ and occurs at about $232^{\circ}$ from the initial direction 


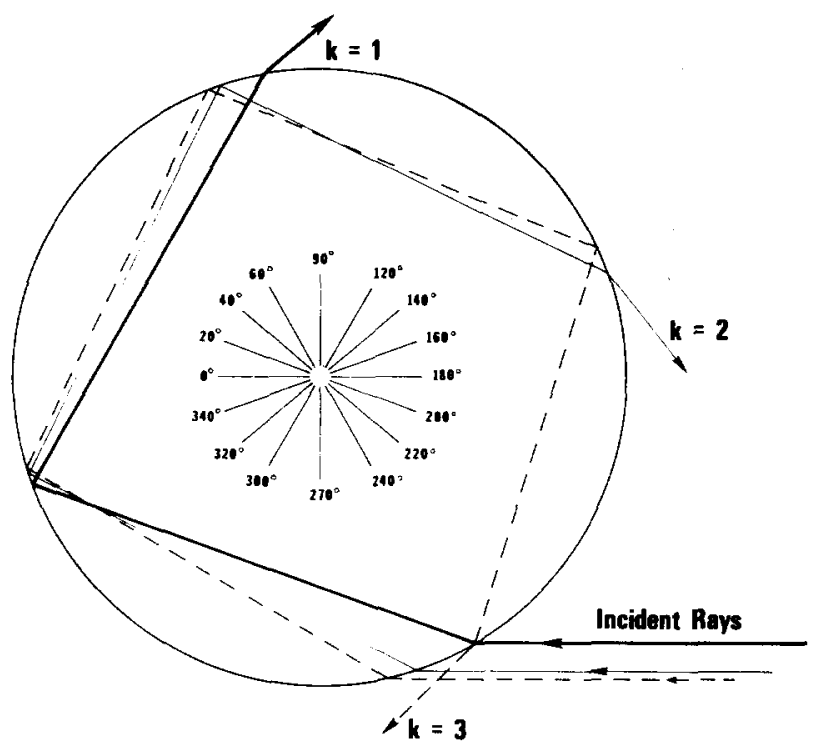

Fig. 5. Cartesian rays for the red light in the first three rainbows are shown. A fully illuminated drop also has a set of these rays symmetric about the $0^{\circ}-180^{\circ}$ axis of the figure.

of the rays.

For the general case of $k$ internal reflections, the total deviation of a ray is

$$
\theta=k 180^{\circ}+2 i-2 r(k+1) \text {. }
$$

The incident angle $i$ corresponding to the minimum deviation after $k$ internal reflections is

$$
\cos i_{k}=\left[\left(n^{2}-1\right) / k(k+2)\right]^{1 / 2} .
$$

For a particular choice of $k$, the rainbow angle $\theta_{c}$ and the Cartesian ray for any of the colors can be found just as they were determined for $k=1$. The Cartesian rays for red light incident on a half-illuminated water drop are illustrated in Fig. 5 for $k=1,2$, and 3 and in Fig. 6 for

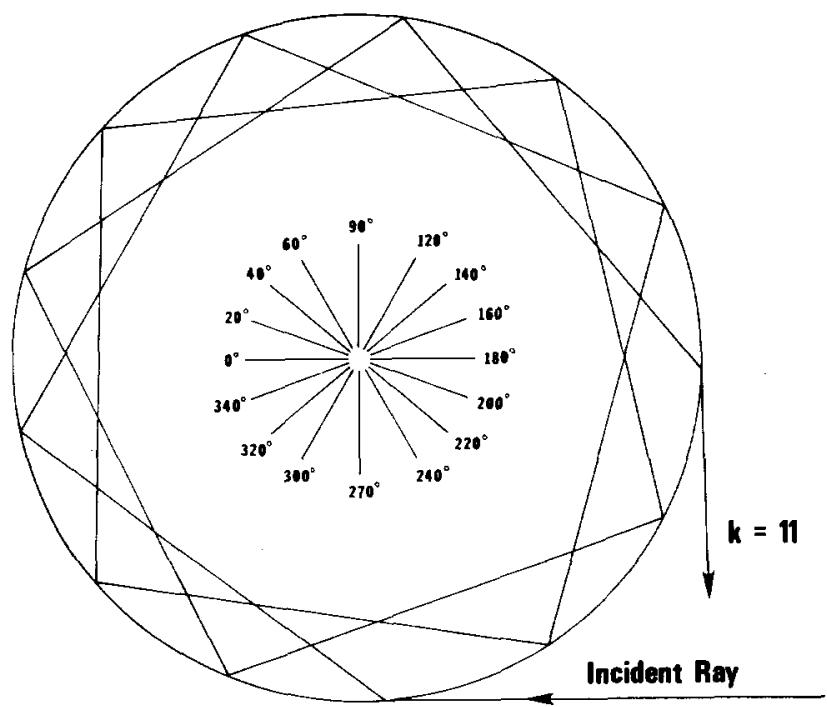

Fig. 6. Cartesian ray for the red light of the 11 th-order rainbow is shown. The symmetric distribution of rays stated in Fig. 5 applies here also. $k=11$. Table $\mathrm{I}$ lists the angular positions and angular widths for the first 20 rainbows for water, using $n=1.331$ for red light and $n=1.343$ for blue light. These results are given in Fig. 7 as a roselike distribution of colors about the drop. ${ }^{13}$ For clarity, the rainbows are shown in the figure as if only half the drop is illuminated. For a fully illuminated drop, the rainbows would be symmetrically placed about a line through the drop's center and parallel to the incident rays. Such a fully illuminated drop would therefore have several of the first twenty rainbows overlapping other rainbows and, in the case of $k=12$, even overlapping itself. The individual colors in Fig. 7, which are only approximately placed within the rainbows, illustrate the color sequence. The full angular widths of the rainbows are accurately drawn using the refractive indices given above for red and blue light.

Were all twenty rainbows visible in the natural landscape, a brief rain on an otherwise sunny day could leave the sky in a dazzling splash of colors as suggested by Fig. 8. Each of the rainbows in the figure would be an arc into and out of the page similar to the commonly seen arcs of the first two rainbows. The rays of sunlight are incident along the horizontal axis, corresponding to a sun at the horizon. For a sun higher in the sky, the distribution of the rainbows would shift counterclockwise. The internal scattering of the Cartesian rays for the first and third rainbows is indicated. As in Fig. 7, the individual colors are only approximately placed but the total rainbow widths are accurately drawn using the red and blue indices of refraction. The circular distribution of the rainbows in Fig. 8 should not be interpreted as suggesting the drops giving the rainbows are at any particular distance from the observer. Only the angular positions can be determined, the drops being anywhere from a few inches to several miles from the observer.

As beautiful as it might be, Fig. 8 is nonetheless fictitious since only two rainbows are ever seen in the natural sky. The first reason for the elimination of the rain-

Table I. The first 20 rainbows of water calculated from geometrical op-

\begin{tabular}{|c|c|c|c|c|c|c|c|c|}
\hline \multirow[b]{2}{*}{$k$} & \multicolumn{2}{|c|}{$\theta_{c}$} & \multirow[b]{2}{*}{$\Delta \theta$} & \multicolumn{2}{|c|}{$i$} & \multicolumn{2}{|c|}{$r$} & \multirow{2}{*}{$\frac{E_{k}\left(10^{-4}\right)}{\text { red }}$} \\
\hline & red & blue & & red & blue & red & blue & \\
\hline 1 & 137.63 & 139.35 & 1.72 & 59.53 & 58.83 & 40.36 & 39.58 & 914 \\
\hline 2 & 230.37 & 233.48 & 3.11 & 71.91 & 71.52 & 45.57 & 44.93 & 390 \\
\hline 3 & 317.52 & 321.89 & 4.37 & 76.89 & 76.62 & 47.03 & 46.42 & 215 \\
\hline 4 & 42.76 & 48.34 & 5.58 & 79.67 & 79.46 & 47.66 & 47.06 & 136 \\
\hline 5 & 127.08 & 133.86 & 6.78 & 81.46 & 81.28 & 47.99 & 47.39 & 94 \\
\hline 6 & 210.90 & 218.86 & 7.96 & 82.72 & 82.57 & 48.18 & 47.59 & 69 \\
\hline 7 & 294.41 & 303.55 & 9.14 & 83.65 & 83.51 & 48.31 & 47.72 & 53 \\
\hline 8 & 17.71 & 28.02 & 10.31 & 84.36 & 84.25 & 48.39 & 47.80 & 42 \\
\hline 9 & 100.86 & 112.35 & 11.49 & 84.94 & 84.83 & 48.45 & 47.87 & 34 \\
\hline 10 & 183.92 & 196.57 & 12.65 & 85.40 & 85.31 & 48.49 & 47.91 & 28 \\
\hline 11 & 266.89 & 280.71 & 13.82 & 85.79 & 85.70 & 48.53 & 47.95 & 23 \\
\hline 12 & 349.81 & 4.79 & 14.98 & 86.11 & 86.03 & 48.55 & 47.97 & 20 \\
\hline 13 & 72.68 & 88.83 & 16.15 & 86.39 & 86.32 & 48.58 & 47.99 & 17 \\
\hline 14 & 155.51 & 172.82 & 17.31 & 86.64 & 86.57 & 48.59 & 48.01 & 15 \\
\hline 15 & 238.31 & 256.79 & 18.48 & 86.85 & 86.78 & 48.61 & 48.02 & 13 \\
\hline 16 & 321.09 & 340.73 & 19.64 & 87.03 & 86.97 & 48.62 & 48.04 & 12 \\
\hline 17 & 43.84 & 64.65 & 20.81 & 87.20 & 87.14 & 48.63 & 48.05 & 10 \\
\hline 18 & 126.58 & 148.55 & 21.97 & 87.35 & 87.29 & 48.63 & 48.05 & 9 \\
\hline 19 & 209.30 & 232.43 & 23.13 & 87.48 & 87.43 & 48.64 & 48.06 & 8 \\
\hline 20 & 292.02 & 316.31 & 24.29 & 87.60 & 87.55 & 48.65 & 48.07 & 8 \\
\hline
\end{tabular}
tics. (Angles are in degrees.) 
bows for $k \geqslant 3$ involves the reduced intensity of the light yielding such rainbows. Since the Cartesian rays strike at greater incident angles for larger values of $k$ (Figs. 5 and 6 , Table I), they are closer to the edge of a drop, with a resulting reduction in the cross-sectional area intercepting the rays. Consequently, the intensity of the emerging cluster of rays about the cartesian ray, is decreased, and the rainbow will be dim. Second, the higher-order rainbows are wider than the first two (Table I, Figs. 7 and 8 ), and the greater dispersion of their Cartesian rays reduces the brightness. Finally, since some light is lost to refraction at each internal reflection, the higher-order rainbows are dimmer because of the greater number of reflections.

If these were the only reasons for the dimness of the higher-order rainbows, keen eyesight might still pick out at least a few of them. None are visible in nature, however, since they are dimmer than at least one of the following: the background sky, the light reflected from the outer surface of the drops [Fig. 1(a)], or the light that traverses and leaves the drops with no internal reflections [Fig. 1(b)]. The outer reflected light is called the direct glare $(G)$ and the light emerging with no internal reflections, the transmitted glare $(T)$. In the experiment described in Sec. VII, the first 13 rainbows are observable because the effects masking natural rainbows are either eliminated or partially removed.

The dark band lying between the primary and secondary rainbows (Fig. 9) has been extensively noted in the literature ${ }^{18}$ The raindrops in this band cannot scatter $k=1$ or $k=2$ rays to the observer. The observer does see $k=1$ light inside and beneath the primary rainbow, but a $k=1$ ray cannot come from the dark band because to do so it would have to be scattered less than the angle of minimum deviation, the Cartesian rainbow angle for $k=1$ rays. By identical reasoning, $k=2$ rays can be seen inside and above the secondary rainbow, but $k=2$ rays from the dark band would require scattering at less than their angle of minimum deviation, the Cartesian angle of the secondary rainbow. Hence, the sky between the primary and secondary rainbows has only glare from the raindrops and background sky light and is therefore darker than the sky beneath the primary rainbow, which also has $k=1$ rays, and the sky above the secondary rainbow, which also has $k=2$ rays. The dark band is prominent in most double rainbow photographs ${ }^{19}$ and in many rainbow paintings and is easily seen and photographed in the experiment of this paper (Fig. 24).

\section{INTENSITY OF SCATTERED LIGHT}

For a particular number of internal reflections, the scattered light is brightest at the angle of the rainbow. In calculating the scattered intensity using geometrical optics, three effects must be investigated. One is the reduction in intensity of the rays with each refraction and reflection. The others are the interception of the incident intensity by the drop and the spreading of the light upon emerging from the drop.

With each refraction and reflection in a drop, the rays of light are reduced in intensity. If a ray is reflected from a surface, the fraction of the incident intensity reflected is

$$
[\sin (i-r) / \sin (i+r)]^{2}
$$

if the ray is polarized parallel to the plane of incidence, or

$$
[\tan (i-r) / \tan (i+r)]^{2}
$$

if the ray is polarized perpendicular to the plane of incidence. The fraction of the incident intensity refracted through the surface is

$$
1-[\sin (i-r) / \sin (i+r)]^{2}
$$

for parallel polarization, and

$$
1-[\tan (i-r) / \tan (i+r)]^{2}
$$

for perpendicular polarization.

If a ray enters a drop and undergoes $k$ reflections, the fraction of the original intensity remaining in the final emerging ray is

$$
E_{k 1}=\left[1-\left(\frac{\sin (i-r)}{\sin (i+r)}\right)^{2}\right]^{2}\left(\frac{\sin (i-r)}{\sin (i+r)}\right)^{2 k}
$$

for parallel polarization, and

$$
E_{k 2}=\left[1-\left(\frac{\tan (i-r)}{\tan (i+r)}\right)^{2}\right]^{2}\left(\frac{\tan (i-r)}{\tan (i+r)}\right)^{2 k}
$$

for perpendicular polarization. The total fraction is

$$
E_{k}=E_{k 1}+E_{k 2} \text {. }
$$

Table I lists the results of these calculations for the red Cartesian rays of the first 20 rainbows. The results for blue rays are almost the same.

One might think that for a particular value of $k$ the $E_{k}$ would be greatest for the Cartesian rays since the scattered light is brightest at the rainbow angles. However, this conclusion is wrong. For example, the values of $E_{1}$ for red and blue $k=1$ rays are given in Fig. 10 for all incident and exit angles. The term $E_{1}$ is not maximum at the primary rainbow but for rays which are incident at $80^{\circ}$, not the $59^{\circ}$ of the Cartesian rays, and which exit near $\theta=150^{\circ}$, not $140^{\circ}$. Hence, the primary rainbow is the brightest of the $k=1$ scattered light in spite of, rather than due to, $E_{1}$.

Although the brightening can be attributed qualitatively to the bunching of the rays at the rainbow angle, the next step would be to calculate the intensity explicitly. ${ }^{20}$ If a thin pencil of light of intensity $I_{0}$ is incident on a drop at polar angles of $i$ and $\phi$ and within a range of angles $d i$ and $d \phi$, the surface element intercepted has an area $a^{2} \sin i d i d \phi$ and the flux of energy at the surface element is

$$
I_{0} \cos i\left(a^{2} \sin i d i d \phi\right)
$$

A fraction $E_{k}$ of the incident energy survives whatever the number of internal reflections $k$ one wishes to consider. This remaining light finally emerges in a solid angle of $\sin \theta d \theta d \phi$ at an exit angle of $\theta$. At a distance $r$ from the drop, the emerging energy is spread over an area of $r^{2}$ times the solid angle. The emerging intensity at $r$ is therefore 


$$
\begin{aligned}
I_{k} & =\frac{E_{k} I_{0} a^{2} \cos i \sin i d i d \phi}{r^{2} \sin \theta d \theta d \phi} \\
& =0.5\left(I_{0} \frac{a^{2}}{r^{2}}\right) E_{k} \frac{\sin (2 i)}{\sin \theta}\left(\frac{d \theta}{d i}\right)^{-1} .
\end{aligned}
$$

The $d \theta / d i$ can be obtained from (3) as

$$
\frac{d \theta}{d i}=2-2(k+1) \frac{\cot i}{\cot r}
$$

Let

$$
D_{k}=0.5 E_{k}\left(\frac{\sin (2 i)}{\sin \theta}\right)\left(\frac{d \theta}{d i}\right)^{-1},
$$

and then

$$
I_{k}=I_{0} a^{2} D_{k} / r^{2},
$$

where only $D_{k}$ depends on the scattering angles.

The term $D_{1}$ for $k=1$ scattering is given in Fig. 11 for red and blue light. The scattering intensity becomes infinite at the Cartesian angles for the two colors because $d \theta / d i=0$ there. This factor of $d \theta / d i$ is therefore the reason the scattered light is brightest at the rainbow angles. In fact, the term is so dominant that the shape of the $E_{1}$ curve of Fig. 10, with its peak near $\theta=150^{\circ}$, is lost in the $D_{1}$ curve of Fig. 11 .

\section{DISPERSION}

The angular dispersion of the light is not the greatest at the rainbow angles. Granted the emerging light is weaker at other angles, one may wonder why some color is not seen at the other angles, especially if the dispersion is greater. Such a consideration is important in this paper's experiment since single drops are examined from less than $0.5 \mathrm{~m}$ and the light emerging at the other angles is clearly seen as individual spots on the drop.

The dispersion can be written

$$
\frac{d \theta}{d \lambda}=\frac{d \theta}{d n} \frac{d n}{d \lambda}
$$

where $d n / d \lambda$ depends on the nature of the scattering material and $d \theta / d n$ depends on the geometry of the scattering. From (3) and Snell's law, the geometrical term is found to be

$$
\frac{d \theta}{d n}=\frac{2(k+1) \sin i}{\left(n^{4}-n^{2} \sin ^{2} i\right)^{1 / 2}}
$$

for given incident angles and $k$ internal reflections. The apparent linear dependence of $d \theta / d n$ on $k$ in (18) may not seem consistent with the rainbow widths $\Delta \theta$ in Table $I$. The widths do not increase linearly with $k$ because the incident angles $i$ for the Cartesian rays are different for each value of $k$ and for each color of light. The term $d \theta / d n$ of (18) depends on both $k$ and $i$. Consequently, not only are the higher order rainbows wider because of the greater number of internal reflections, but also because their Cartesian rays are incident at greater angles (Fig. 12). Using (4) for the incident angles of the Cartesian rays, the term $d \theta / d n$ for the Cartesian rays is found to be

$$
\left(\frac{d \theta}{d n}\right)_{c}=\frac{2}{n}\left(\frac{(k+1)^{2}-n^{2}}{n^{2}-1}\right)^{1 / 2}
$$

One color will dominate the others if the drop is observed at that color's $\theta_{c}$. At the $\theta_{c}$ for blue light, the emerging light has a mixture of all visible colors, but because the blue rays are bunched at that angle, blue dominates. At the $\theta_{c}$ for red light the situation is simpler. The red is prominent at its $\theta_{c}$ because of the bunching and also because there is no competition from the other colors. Since red light has the smallest index of refraction in the visible spectrum, it experiences the smallest angle of minimum deviation. For example, in the primary bow $\theta_{c}$ is $137.63^{\circ}$ for the red and $139.35^{\circ}$ for the blue. Hence, when an observer is at the $\theta_{c}$ for red, no blue light is in the emerging light (Fig. 11).

At most angles other than the rainbow angles, the light emerging from the drop is white. The lack of color separation may seem strange, especially if the rays enter the drop at incident angles greater than that of the Cartesian rays and therefore suffer greater dispersion (Fig. 12). For instance, if a drop is viewed from the direction of the emerging ray $h$ in Fig. 2, the emerging light will be white even though it has suffered greater dispersion than the Cartesian ray $f$ of the figure. In viewing from the direction of ray $h$, all the colors from the drop are seen simultaneously, each slightly but imperceptibly displaced at its exit point on the drop. The relative intensity of light suffering an internal reflection and a deviation of $\theta$ is given in Fig. 11. For ray $h$, which is incident at $i=80^{\circ}$ and which exits at about $\theta=150^{\circ}$, all colors have about the same intensity and therefore combine to give white light. Experimentally, the spot of emerging light resulting from ray $h$ can be distinguished on the drop and confirmed to be white. For smaller incident angles, except for those near the Cartesian rays, the emerging light always has about equal intensities of the colors and is therefore white.

There is one exception to this argument, however. If the drop is viewed from the direction of the emerging rays that have been incident nearly tangentially to the drop, i.e., at $i=88^{\circ}-90^{\circ}$, the emerging light is blue. The blue light is weak but can be seen if other spots of light on the drop are eliminated. The light is blue because the other colors, especially red, may be many times weaker than the blue, as indicated in Fig. 11 in the range of $\theta=162^{\circ}-167^{\circ}$ for the part of the curves corresponding to $i=88^{\circ}-90^{\circ}$. Blue is refracted more than red by water. For the extreme case in which white light is incident at $i=90^{\circ}$, the blue is deviated a few degrees more than the red (Fig. 13). Since the upper limit of $\theta$ is about $165.0^{\circ}$ for red light and about $167.5^{\circ}$ for blue light, the emerging light will give a blue spot on the drop if the drop is viewed at $166^{\circ}$.

Bright, white light also emerges at $166^{\circ}$ from rays incident at $14^{\circ}$ and normally masks the blue spot. If falling raindrops are viewed at a scattering angle of $166^{\circ}$, they are white and give no hint of blue. The blue can be distinguished from the brighter white light if the observer is within about $30 \mathrm{~cm}$ of a drop since the blue light emerges from the edge of the drop and the white light more toward the center of the drop.

Similar considerations can be made for $k>1$ rays. Again, the emerging light is white except at the angles of the rainbows, where all colors are distinguishable, and at 


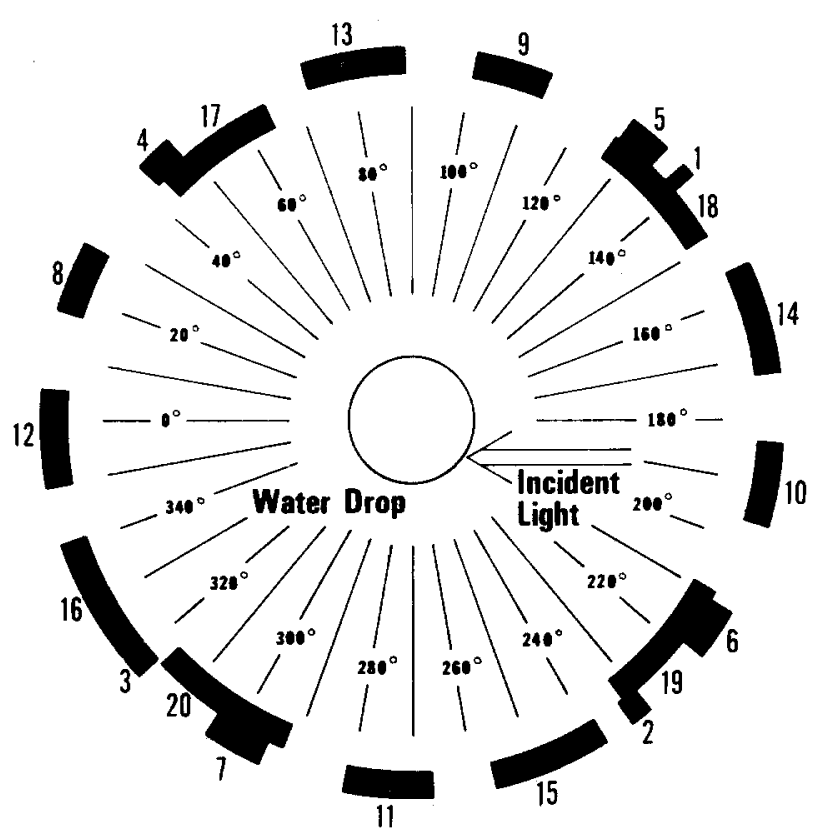

Fig. 7. There is a roselike distribution of rainbows in the scattering plane of a water drop.

the portion of the curves for nearly tangential incidence, where the emerging light is blue. There is also a slight blue tinge to the nearly tangentially incident light that crosses and exits from a drop with no internal reflections [Fig. 1(b)].

\section{RAINBOWS FROM OTHER REFRACTIVE INDICES}

Since the Cartesian rays of the rainbows depend on the refractive index, a transparent drop with a refractive index different from water will have rainbows shifted from those of water. ${ }^{11,21}$ These shifts are described in the experimental section of this paper for two liquids: Karo light corn syrup $(n=1.47)$ and diodomethane $(n=1.75)$.

The Cartesian angles $\theta_{c}$ for the first thirteen rainbows are plotted in Figs. 14(a) and 14(b) as functions of $n$. The drop was assumed to be only half illuminated as in Figs. 2-7. A fully illuminated drop would have curves symmetric about $180^{\circ}$ in Figs, 14(a) and 14(b). The curves are steepest in the figure for the low values of $n$ near the $n=1$ of air, and as $n$ increases, the curves become less steep. The $k=1$ curve has a slope of zero for $n=2$, giving a Cartesian ray emerging at $180^{\circ}$. This condition is the theoretical limit of the $k=1$ rainbow, ${ }^{21}$ for it corresponds to light rays incident at $i=0^{\circ}$ (Fig. 15), that is, directly at the center of the drop, and the rays are backscattered directly toward the light source. These rays experience no dispersion, as indicated by the $k=1$ curve at $\theta=180^{\circ}$ in Fig. 16, which is based on the dispersion relationship of (19) for the Cartesian rays. With no dispersion there can be no rainbow. Hence, there is no longer a $k=1$ rainbow for $n>2$. All the other rainbows, for $k>1$, meet similar fates ${ }^{21}$ for greater values of $n$.

\section{POLARIZATION}

For water the rainbows appear to be nearly completely polarized parallel to the plane of incidence. The polarization sense is noted in some textbooks ${ }^{22}$ and can easily be checked with a natural rainbow and a polarized filter such as in polarized sunglasses. Figure 17 is a graph of

$$
P=E_{k 1} /\left(E_{k 1}+E_{k 2}\right)
$$

for the range of $n$ of interest in the experiment of this paper. For water there is only a small difference in polarization between the $k=1$ and $k=2$ rainbows. For greater values of $n$, the $k>1$ rainbows remain parallel polarized up to $n=2$. The $k=1$ rainbow, however, decreases in polarization and is unpolarized at $n=2.0$ where the rainbow disappears. The polarization of the rainbows depends on $n$ only because $E_{k 2}$ does. Using (9), $E_{k 1}$ can be reduced to

$$
E_{k 1}=\left(1-q^{2}\right)^{2} q^{2 k}
$$

where

$$
q=\left[\left(k^{2}+k+1\right)^{1 / 2}-1\right] /\left[\left(k^{2}+k+1\right)^{1 / 2}+1\right] .
$$

Since there is no dependence on $n$ in (21), $E_{k 11}$ is independent of $n$.

\section{EXPERIMENTAL GEOMETRIC RAINBOWS}

Higher-order rainbows up to $k=13$ can be observed from an individual water drop in the lab under relatively simple conditions. Water is sprayed onto a thin wire hanging from a ringstand. The wire is coated with Apiezon wax to blacken the wire and to facilitate collect-

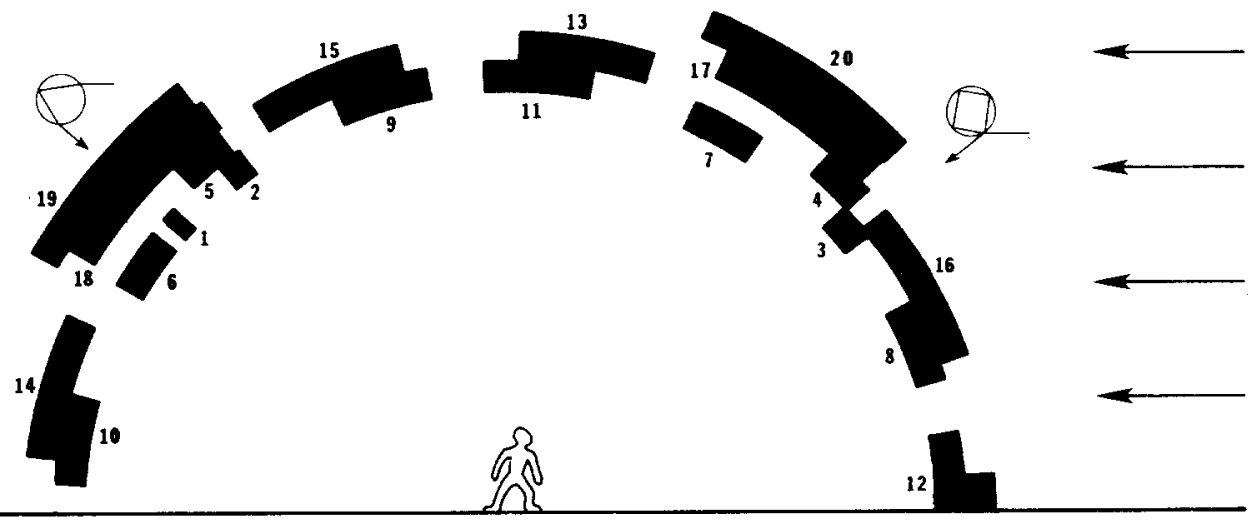

Fig. 8. Angular distribution of the first 20 rainbows of raindrops are shown as they would be seen in the natural landscape. 

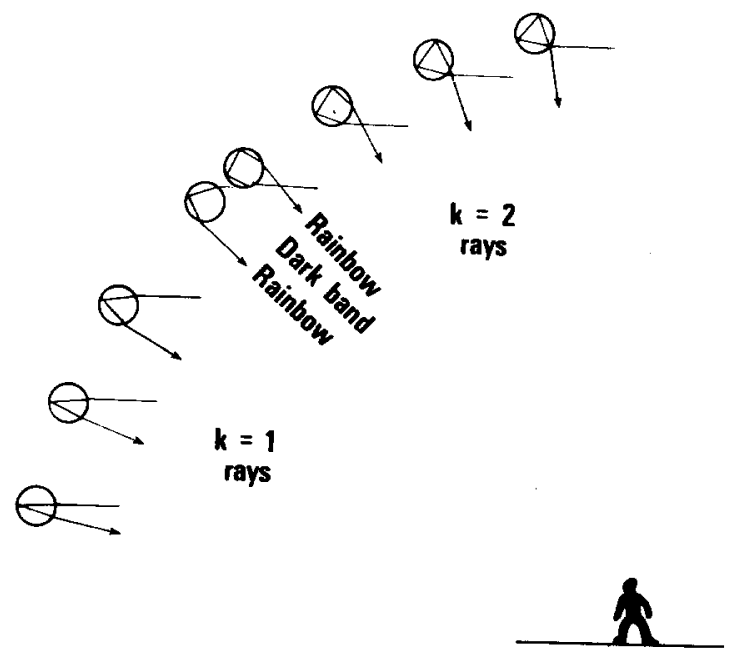

Fig. 9. An observer sees white $k=1$ rays below the primary rainbow and white $k=2$ rays above the secondary rainbow. Neither $k=1$ or $k=2$ rays can originate between the two rainbows, and consequently that area of the sky is relatively dark.

ing and maintaining a drop at the end of the wire. Ideally, the drop should touch the wire only at the top of the drop.

Once the drop is suspended, a student spectrometer ${ }^{23}$ is pushed under the drop so that the drop is over the center of the spectrometer and can be viewed through the telescope arm (Fig. 18). Light from a lamp or laser is directed through the collimating arm and onto the drop. A card is used to block the light from the wire itself so as to minimize stray light from the drop area. Lowering a large cardboard box over the spectrometer and turning off the room light eliminates most of the background light, and only the drop is then illuminated.

With the apparatus positioned properly, the light rays contributing to the rainbows traverse a circular cross section of the drop. Several of the rainbows are easily recognized with the naked eye by the vivid colors on the drop, and their angular positions can be measured accurately if the telescope has cross hairs. To determine from what part of the drop the colors are emerging, the drops should be viewed with the naked eye or through the tele-

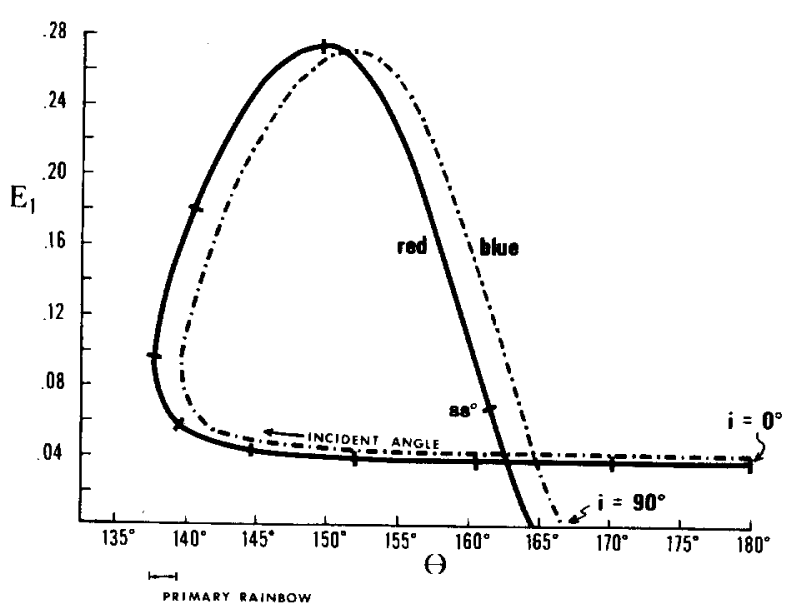

Fig. 10. $E_{1}$, given in (11), is the fraction of the incident energy surviving in the $k=1$ rays. Values are shown for both red and blue light. The incident angles increase in the direction of the arrow and are marked at $10^{\circ}$ intervals except for $i=88^{\circ}$, which is also indicated.

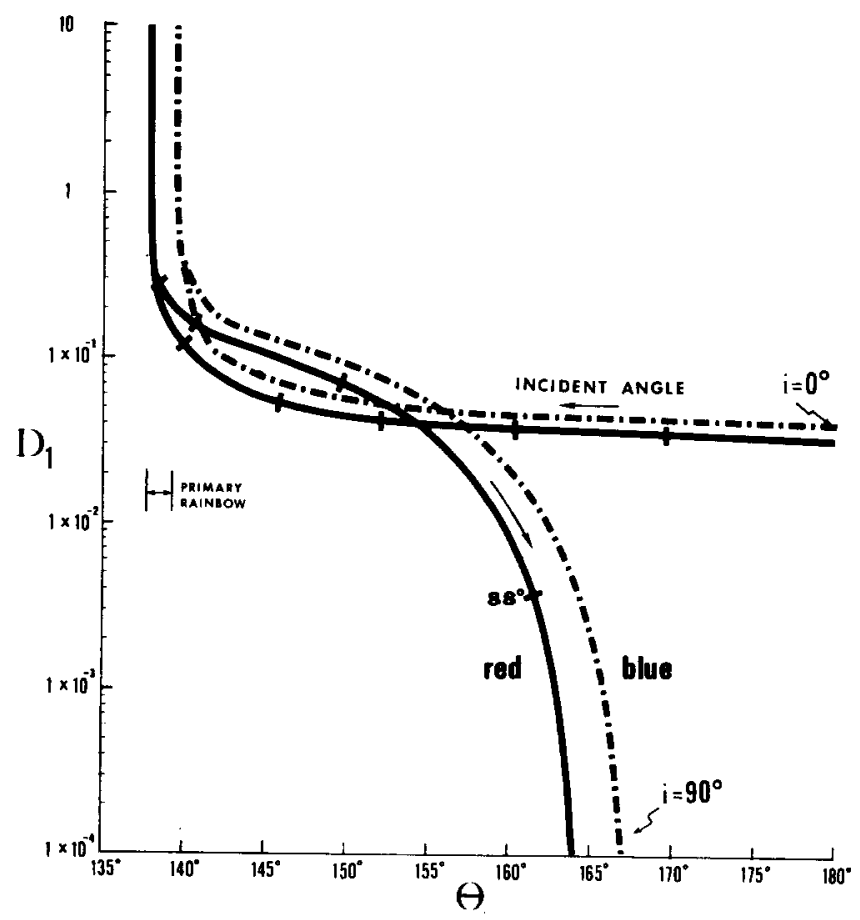

Fig. 11. $D_{1}$ is related to the scattering intensity of the $k=1$ rays by (15). Values are shown for both red and blue light. The incident angles increase according to the arrows and are marked at $10^{\circ}$ intervals except for $i=\mathbf{8 8}^{\circ}$, which is also indicated.

scope with the eyepiece removed. The advantage in using the telescope tube in this way is that angular readings can be made whereas with naked-eye viewing they could not. This procedure is especially required with the dimmer higher-order rainbows since their appearance on the drop must be distinguished from other, brighter spots of light on the drop.

If the drop is half-illuminated (in the manner indicated in Figs. 2-7) by half-blocking the incident light with the card and if it is then viewed at the angles of the red in the rainbows (Table I), the drop appearances to the naked eye are as given in Fig. 19. The red light of the rainbows is represented by a small spot on the drawings and is

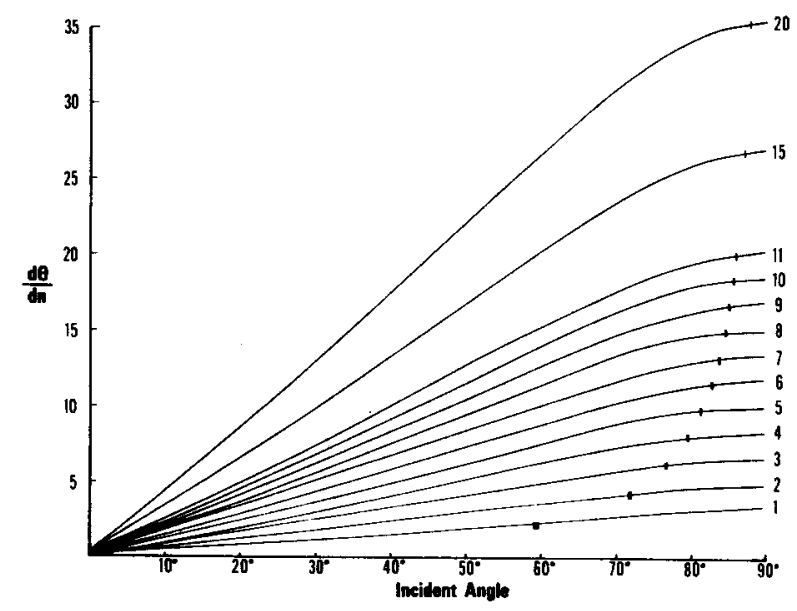

Fig. 12. $d \theta / d n$ is one term in the dispersion relationship of (17). The number of internal reflections is given to the right of the curves. The small rectangles indicate the range of incident angles of the Cartesian rays (Table I). An intermediate value of $n=1.337$ for water is used to calculate $d \theta / d n$. 


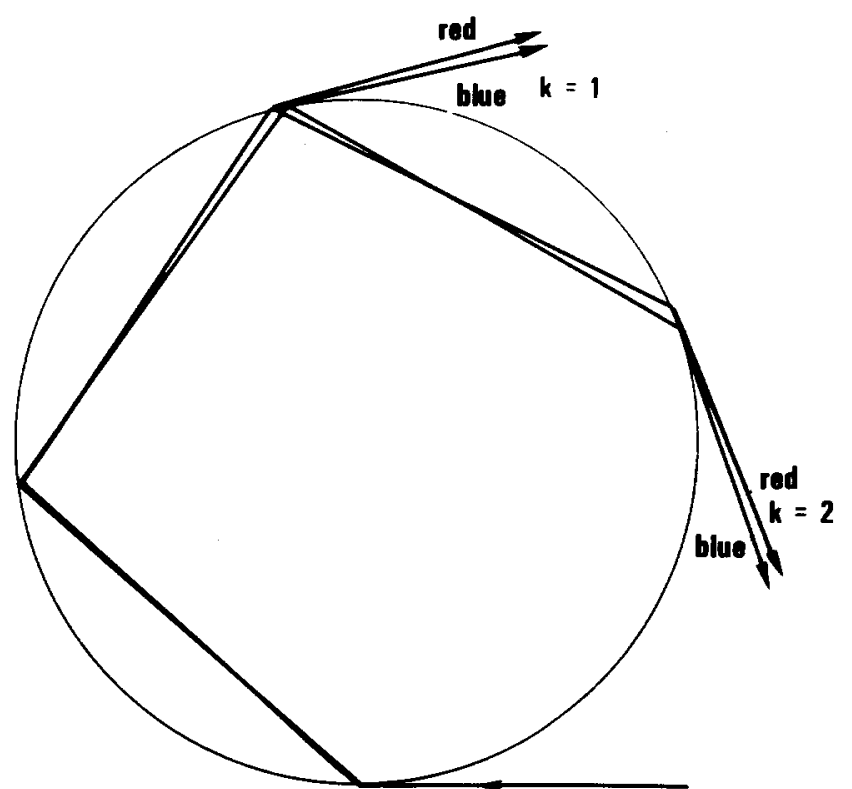

Fig. 13. Blue and red rays resulting from a white ray incident tangentially to the water drop are shown for one and two internal reflections.

labeled $R$. Spots of direct $(G)$ and transmitted $(T)$ glare are also present unless eliminated by a selective blocking of the incident light. The rainbow spots for the higherorder rainbows occur closer to the edge of the drop and are therefore harder to detect. The highest order in water that was easily seen in the laboratory was $k=11$, which appeared as a colored spot at less than $0.5 \%$ of the drop's radius from the edge. The highest order for water that could be observed was $k=13$, which was $0.2 \%$ of the drop radius from the edge.

In order to locate the weaker rainbows, background light and glare must be reduced as much as possible. Even with these measures, the direct and transmitted glare spots are still present and may mask the rainbows. The glare can be partially or completely removed in some cases without altering the rainbows by partially blocking the incident light. For example, the direct glare indicated in the $k=2$ drawing of Fig. 19 can be blocked without affecting the $k=2$ rainbow. A card is inserted as shown in Fig. 18 and slowly brought across the drop toward the observer who is viewing the drop at about $\theta=232^{\circ}$. At some point past midway across the drop, the card blocks those rays reflecting from the outer drop surface to the observer (Fig. 20). With the observer at $\theta=232^{\circ}$, those glare rays must be incident at $26^{\circ}$ to the drop's outer surface so as to reflect to the observer. The rays giving the $k=2$ rainbow are incident at a greater angle to the drop's surface, $72^{\circ}$ (Fig. 5). Since they are therefore incident on the drop closer to the observer, they are not blocked by the card. The result, then, is that the glare rays are eliminated whereas the rainbow rays are not. This technique can always be used to reduce or eliminate glare, both the direct $(G)$ and the transmitted $(T)$ types, when the glare rays are incident at a different spot on the drop than the rainbow rays. The smaller the drop, however, the more difficult it is to properly position the blocking card, so best observations are obtained with larger drops.

The first 13 rainbows from a water drop were seen with this experimental arrangement. Using drops with diameters of about $1.5 \mathrm{~mm}$, most of the rainbow angles

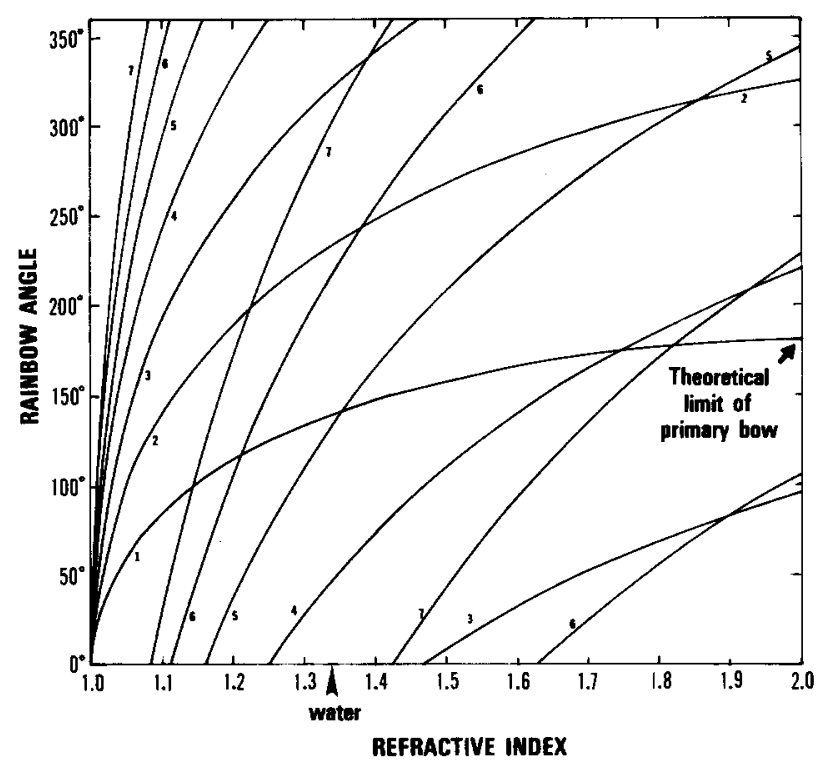

(a)

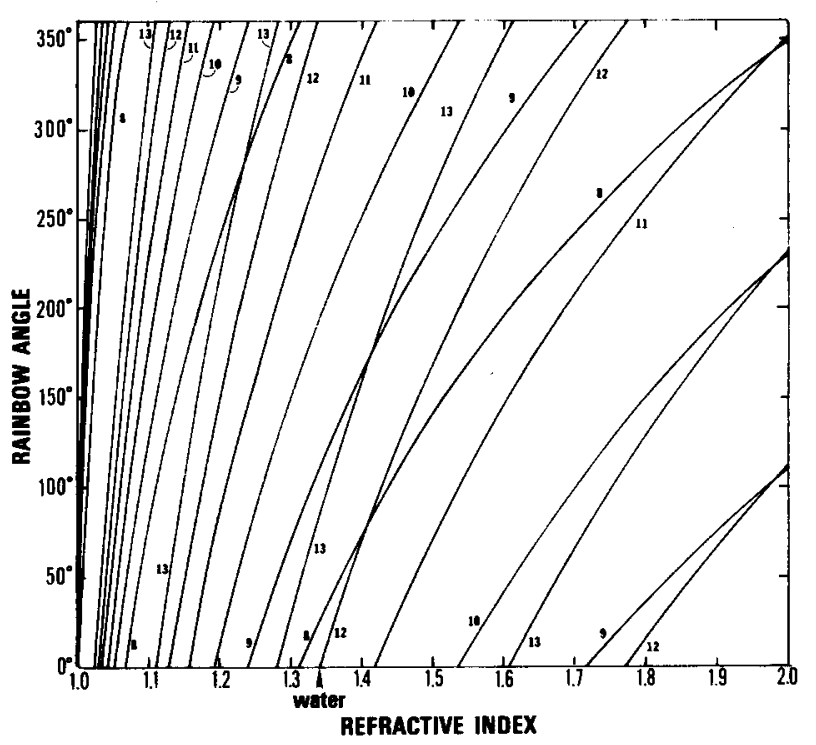

(b)

Fig. 14. Cartesian rainbow angles $\theta_{c}$ for the first 13 rainbows are given as functions of the refractive index. The drop is taken to be halfilluminated as in Figs. 2-7. The angular coordinate system is that in Figs. 5 and 6.

were found to be within a degree of their theoretical values given in Table I. Rainbows $k=8$ and $k=12$ were difficult to locate even with the blocking technique however, because they required looking toward the light source and the direct glare rays $(G)$ were incident at approximately the same angles as the rainbow rays and thus could not be blocked. The $k=11$ rainbow was weak but surprisingly visible even without blocking the direct glare. Only 13 rainbows were seen here, but the next 6 rainbows Billet ${ }^{13}$ saw (1868) in thin, falling streams of water should be observable with this experimental apparatus. One needs keen eyesight, some patience in carefully leveling the incident light beam, and a drop of at least a millimeter in radius so that the blocking technique can be successfully employed.

In most of the 13 rainbows the experimental error can be one degree or less if the rainbow can be distinguished 


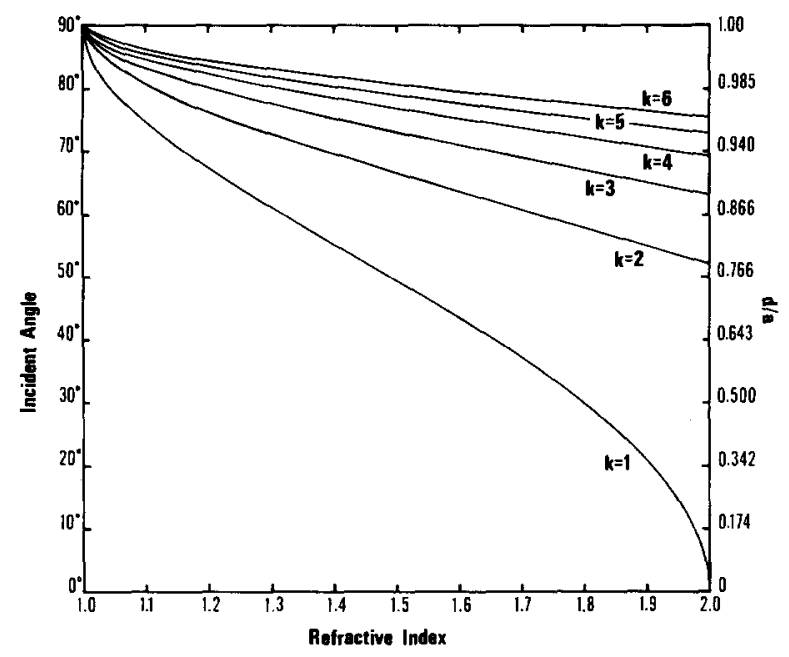

Fig. 15. Incident angle of the first six Cartesian rays. The drop radius is $a$, and $d$ is the pernpendicular distance between the incident Cartesian ray and the ray of normal incidence.

from the glare. Since the $k=1$ and $k=2$ rainbows are relatively bright, the telescope's eye piece with its cross hairs can be used to find the rainbow colors to about an arc minute. The geometrical optics behind the Table I values, however, is approximately correct only for drops with radii larger than $1 \mathrm{~mm}$. With smaller radii there should be significant discrepancies between the Table I locations of the rainbows and the actual experimental locations. These discrepancies, due to the wave interference of light, are discussed in Sec. VIII.

The polarizations of the observed rainbows were checked with a hand-held polarized filter and all were found to be polarized parallel to the plane of incidence. This result approximately confirms Fig. 17 in that such polarization corresponds to $P=1$ there. Apparently much more careful measurements are required to observe the small perpendicular component of the $k \geqslant 2$ scattering in water as indicated in the figure.

If the drop is viewed at angles other than the rainbow angles, several white spots may be seen in addition to the glare spots. For examples, Figs. 21(a) and 21(b) indicate two white spots, $A$ and $B$, if the drop is viewed at $\theta=145^{\circ}[21(\mathrm{a})]$ and $\theta=240^{\circ}[21(\mathrm{~b})]$. These spots are due to rays that are reflecting once [Fig. 21(a)] or twice [Fig. 21(b)], but are not emerging at the rainbow angles.

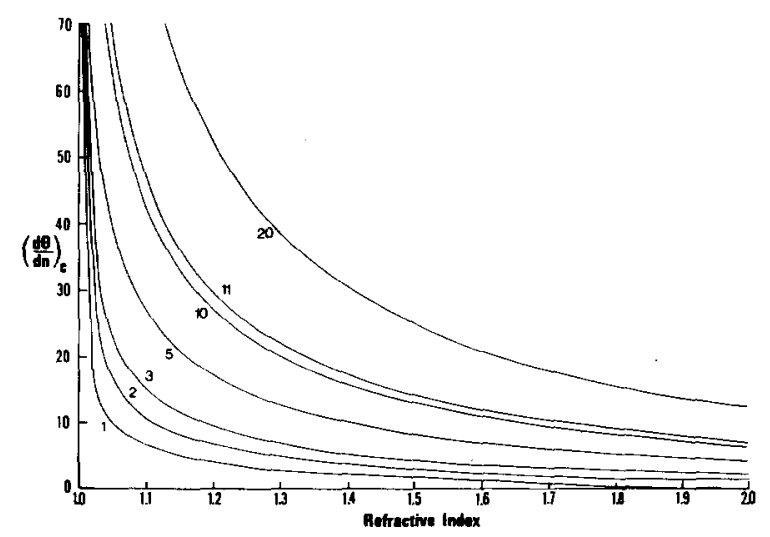

Fig. 16. $(d \theta / d n)_{c}$ is one term (19) in the dispersion of the Cartesian rays. The curves are labeled by the $k$ values.

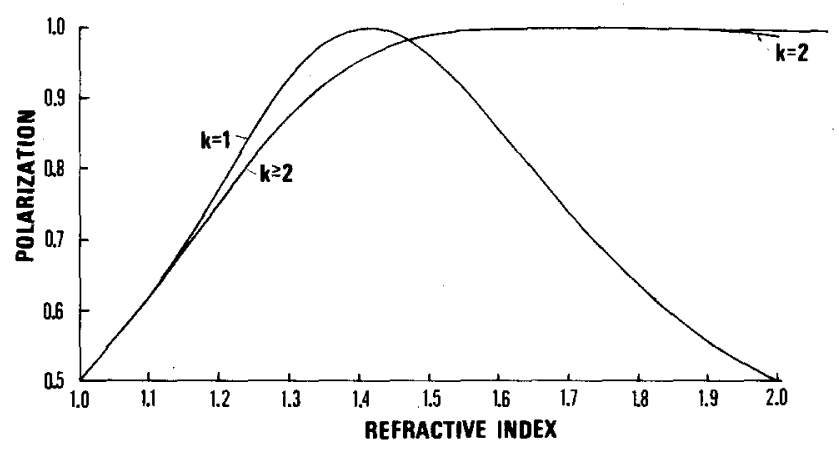

Fig. 17. Polarization as defined in (20).

Spot $A$ of Fig. 21(a) corresponds to ray $d$ in Fig. 2, whereas spot $B$ corresponds to a ray intermediate between rays $g$ and $h$ in Fig. 2. Similarly, spots $A$ and $B$ of Fig. 21 (b) correspond to rays $f$ and $h$ in Fig. 4 . As the viewing angle moves from $\theta=145^{\circ}$ for Fig. 21(a) to the angle of the primary rainbow, $138^{\circ}$, spots $A$ and $B$ move together, finally merging when the rainbow angle is reached, where they become colorful and bright. Similarly, merging of the spots of Fig. 21(b) occurs by moving from the $\theta=240^{\circ}$ of that figure to about $\theta=232^{\circ}$, the angle of the secondary rainbow.

Spots $B$ in both Figs. 21(a) and 21(b) are white even though the rays emerging there suffer greater dispersion than do the Cartesian rays of the primary and secondary rainbows. From the discussion in Sec. IV, these spots are white because approximately equal intensities of the visible colors are emerging (Fig. 11).

Rays incident nearly tangentially to the drop must give blue light if the drop is viewed near $\theta=165^{\circ}$ for $k=1$ scattering. The blueness results because the red light has a slightly smaller $n$ than does blue light (Fig. 13) and is considerably less intense for $\theta>160^{\circ}$ (Fig. 11). Such a blue spot near $166^{\circ}$ is easily seen on the water drop.

Transparent liquid drops with refractive indices differing from that of water give rainbows shifted in $\theta$ as shown in Figs. 14(a) and 14(b). One of the cheapest and easiest alternative fluids is Karo light corn syrup, whose index of refraction is approximately $1.47-1.48$. The syrup can be applied to the end of the wire with a straight pin or paper clip until there is enough fluid to descend and form a drop. Once suspended, the syrup drops can be left to dry. One must be careful, however, that the syrup drop does not dry in a distorted shape. With a syrup drop of about $1 \mathrm{~mm}$ in radius, all of the rainbows up to $k=13$ were seen, except for $k=7$ and 10 which could not be

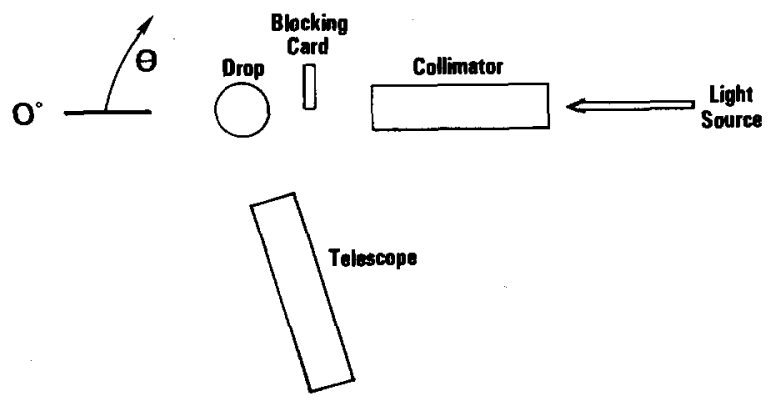

Fig. 18. Apparatus of the scattering experiment as seen in an overhead view. The telescope and collimator are parts of a student spectrometer. 

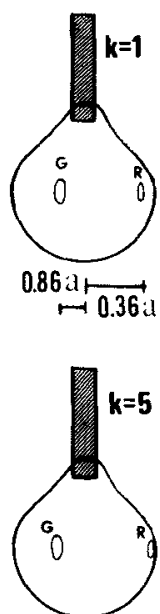

$0.989: \stackrel{\longrightarrow}{0.45 ; 1}$
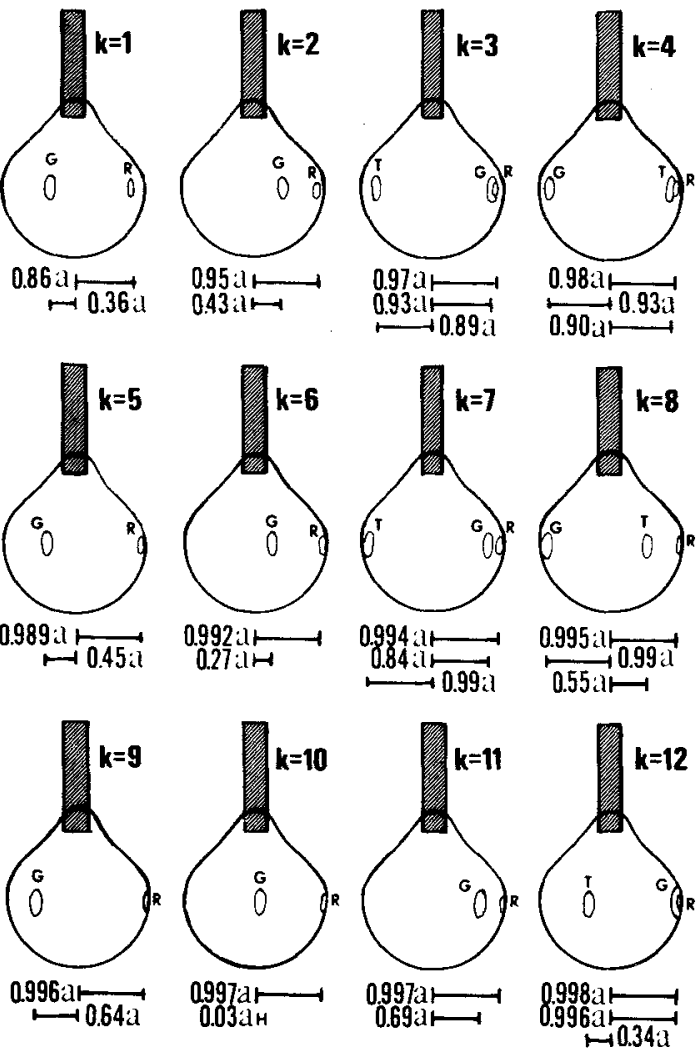

Fig. 19. Water drops are shown as they theoretically would appear when viewed in the scattering plane at the angles of the first 13 rainbows. $R$ represents the red spot of the rainbow; $G$ is the spot of direct glare; and $T$ is the spot of transmitted glare. The spots are located as fractions of the drop's radius $a$.

separated from the glare. In addition, although it was only a tiny speck on the drop, the $k=17$ rainbow was observed. With an appropriate choice of $n$, between 1.47 and 1.48 , the observed positions fall within $1^{\circ}$ or $2^{\circ}$ of the calculated positions. If the drop is left for several days, all the observed rainbows shift considerably as the syrup solution becomes more concentrated and thus has a higher index of refraction. A typical change is $\Delta n \approx 0.025$ in 2 days. The corresponding shift in rainbows can be calculated or taken from Figs. 14(a) and

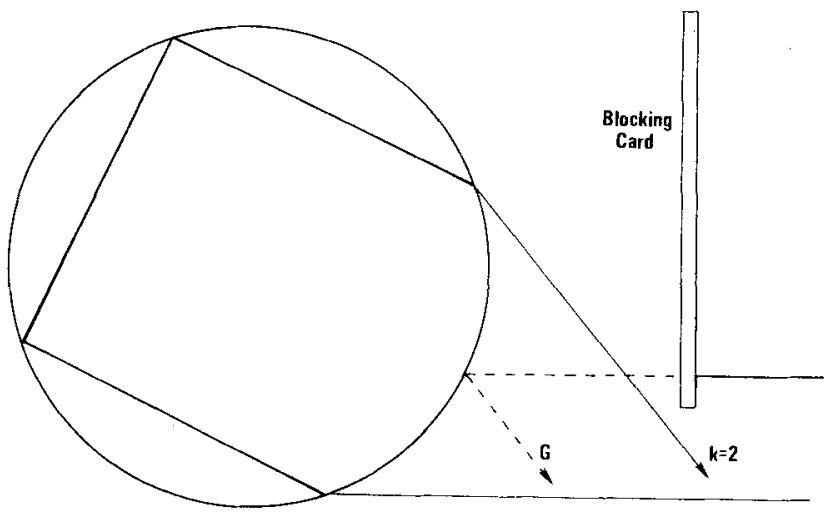

Observer

Fig. 20. Selective blocking of the incident light can eliminate some of the glare rays.

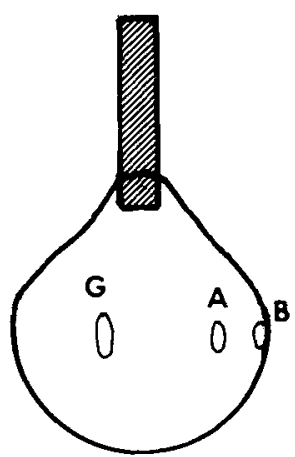

(a)

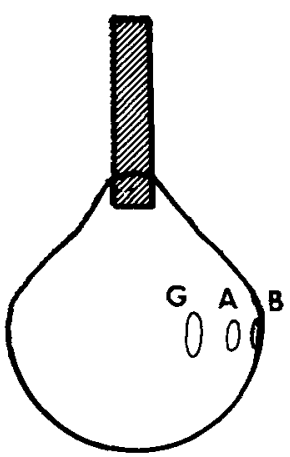

(b)
Fig. 21. Bright spots on the water drop when viewed at $\theta=138^{\circ}$ (a) and $232^{\circ}$ (b).

14(b). No difference could be detected in the polarization of these rainbows; they were all polarized parallel to the plane of incidence as indicated in Fig. 17. (The optical activity of syrup is too small to have an effect here.)

To obtain an even greater index of refraction, a drop of diiodomethane, ${ }^{24}$ which has $n=1.749$ at $\lambda=5890 \AA$, was suspended and the rainbow angles measured. Rainbows $k=1$ and 4 were too close to being directly backscattered to be observed, and rainbows $k=3$ and 9 were apparently lost in glare that could not be blocked. Several other rainbows, $k=2,5,6,7,8$, and 10 , were seen and found to be within a few degrees of their theoretical positions as given in Figs. 14(a) and 14(b). They were also found to be polarized parallel to the plane of incidence as predicted by Fig. 17.

\section{RAINBOWS AS INTERFERENCE PATTERNS}

In observing the rainbow from single drops, two unexpected phenomena occur. As the drops evaporate and decrease in radius, there is a slight shift in the rainbow angles. This shift is not predicted by the geometrical optics of Descartes and Newton, for there is no drop size de-

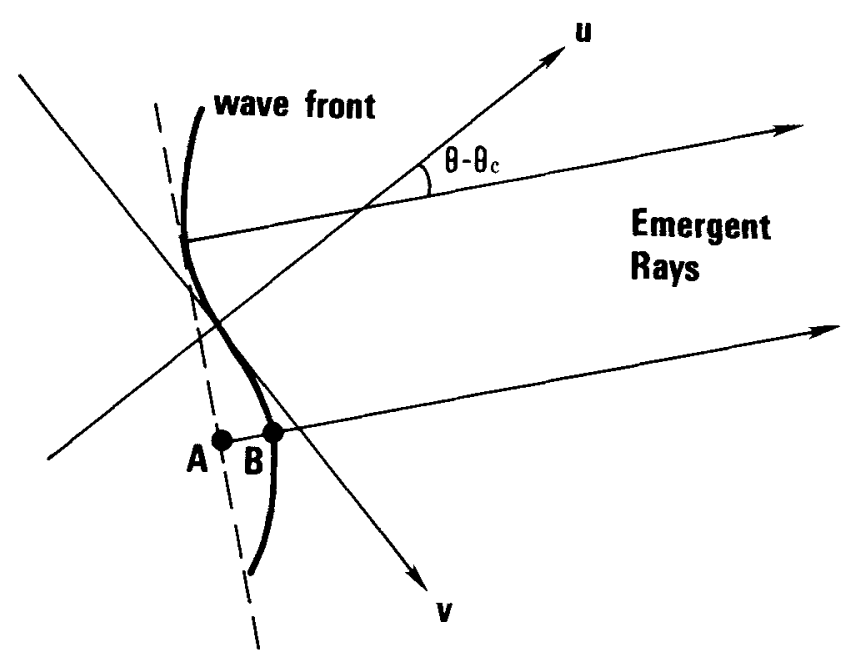

Fig. 22. The wavefront emerging near the rainbow angle is approximately cubic rather than planar. The observer is at angle $\theta$, which is near the primary rainbow angle of $\theta_{c}$. One may superimpose this figure on Fig. 5 such that the $u$ axis lies along the emergent $k=1$ Cartesian ray of Fig. 5 . 


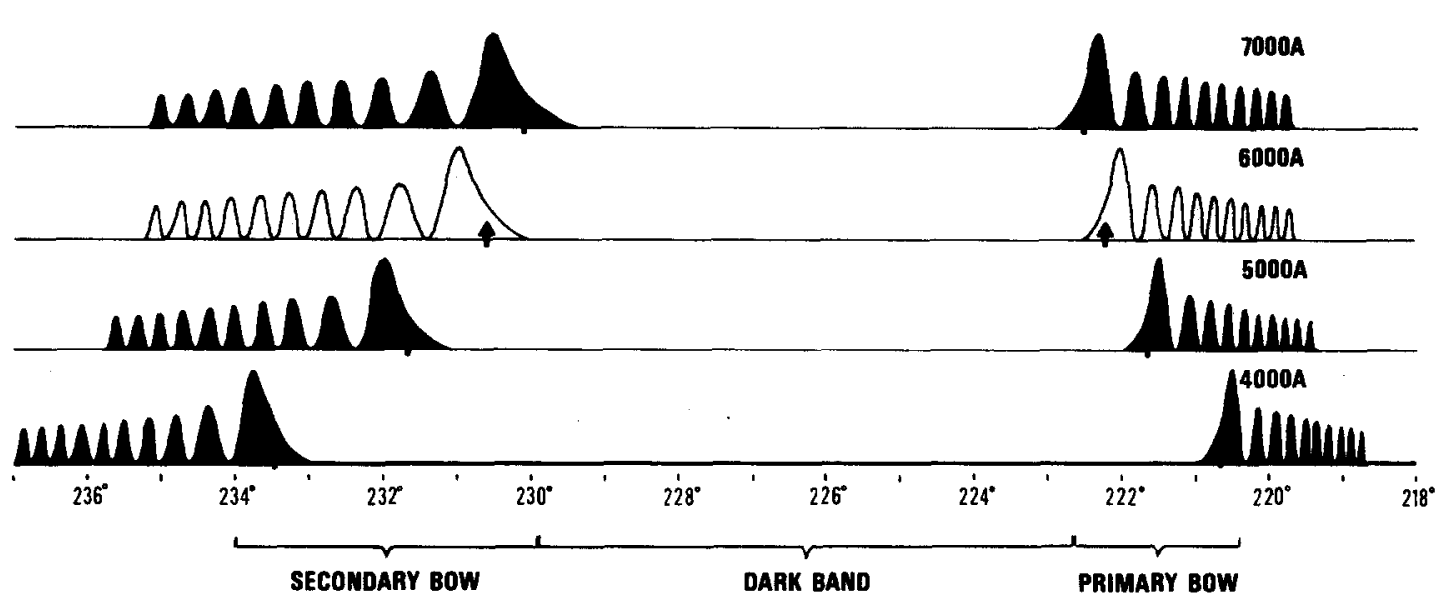

(a)

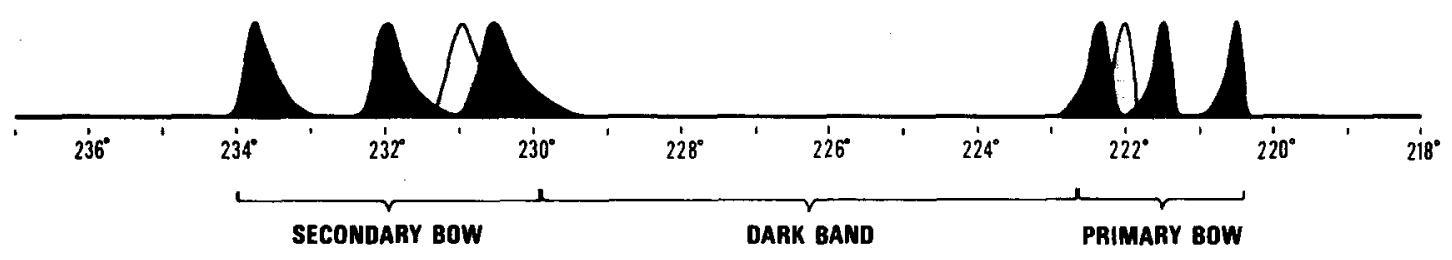

(b)

Fig. 23. (a) The patterns of scattered light for several particular wavelengths are shown for a water drop $(a=1 \mathrm{~mm})$ viewed from $\theta=218^{\circ}-237^{\circ}$ when fully illuminated with an ideal white light source. The Cartesian angles $\theta_{\mathrm{c}}$ are indicated for each wavelength by the vertical arrows. The arrangement of the rainbows shown here is also the same if the horizontal axis is relabeled with $\theta$ increasing to the right and with $236^{\circ}$ replaced by $124^{\circ}$ and the other angles relabeled accordingly. (b) The composite of the principal maxima of (a) illustrates the extent of the primary and secondary rainbows. All intermediate colors would have to be included to simulate the full color of natural rainbows.

pendence in the calculations of $\theta_{c}$. If the drop is illuminated with a single color of light, the pattern of scattered light is also surprising. Rather than a single narrow bright band of light at the theoretical $\theta_{c}$ for that color, there appears an interference pattern having a principal maximum peaking at a slightly greater $\theta$ and having many smaller maxima at even larger $\theta$.

Airy has accounted for these interference patterns by examining the relative phase of the rays emerging near the rainbow angle. ${ }^{25}$ The light emerging near $\theta_{c}$ is not a plane wave even if a plane wave is initially incident on the drop. Since the rays incident on the drop near the cartesian ray have different path lengths inside the drop, they emerge out of phase with each other. Near the rainbow angle the resulting shape of the emerging wave is approximately given ${ }^{26}$ by

$$
u=-h v^{3} / 3 a^{2},
$$

where $u$ and $v$ are coordinates shown in Fig. 22, $h$ is given in (24), and $a$ is the drop radius. The $u$ axis lies along the emerging Cartesian ray for the $k=1$ rainbow of Fig. 5. Included in Fig. 22 are two light rays that will be observed at an angle $\theta$ near the Cartesian rainbow angle $\theta_{c}$ for the primary bow. Those rays are out of phase at the point of observation because of their path difference $A B$ to the observer. The intensity of the resulting interference pattern can be expressed ${ }^{27}$ as

$$
I=4 I_{0}\left[\left(3 a^{2} \lambda / 4 h \cos \left(\theta-\theta_{c}\right)\right]^{2 / 3} f^{2}(z),\right.
$$

where $I_{0}$ is the intensity per unit length of the emerging wavefront, $\theta$ is the angle of observation, and

$$
h=0.75 \sin i / \cos ^{3} i .
$$

The term $f(z)$ is called the rainbow integral and is given as

$$
f(z)=\int_{0}^{\infty} \cos (\pi / 2)\left(z t-t^{3}\right) d t
$$

in which

$$
\begin{aligned}
z & =4 l\left(\theta-\theta_{c}\right) / \lambda, \\
t & =v / l,
\end{aligned}
$$

and

$$
l=\left(3 \lambda a^{2} / 4 h\right)^{1 / 3} \text {. }
$$

The function $f^{2}(z)$ has been tabulated. ${ }^{28}$ For a particular wavelength this function gives bright and dark bands near the Cartesian rainbow angle. Each wavelength in the incident sunlight or artificial light will yield such a pattern, but the principal maxima for the different wavelengths occur at different angles because of dispersion. The patterns for four particular wavelengths are given in Fig. 23(a). What is normally seen in the rainbows is the summation of these interference patterns for all the colors in the solar spectrum, the principal maxima composing what is said to be the natural rainbow. This summation is depicted in Fig. 23(b) for four wavelengths. To imitate the natural double rainbow, one should rotate Figs. 23(a) and 23(b) $90^{\circ}$ clockwise and imagine that the patterns for all the intermediate colors have been included. Then the secondary rainbow is above the primary rainbow, with the 


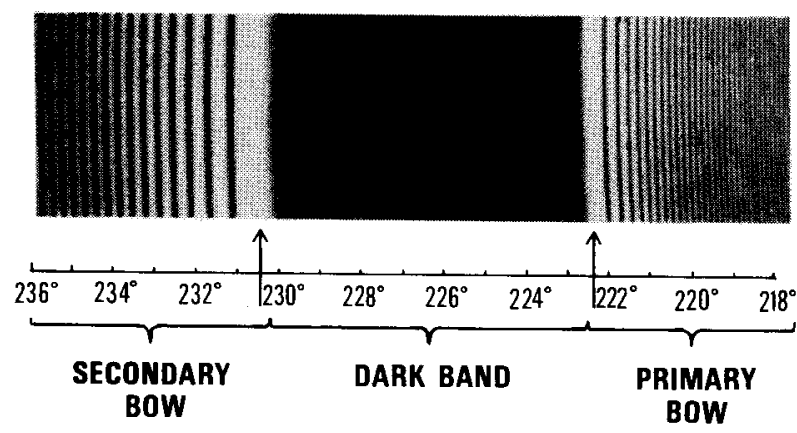

Fig. 24. Interference patterns of the primary and secondary rainbows for the scattering of $\mathrm{He}-\mathrm{Ne} 6328-\AA$ light from a single water drop. The Cartesian angles $\theta_{c}$ are indicated by the vertical arrows. The geometry is the same as in Figs. 23(a) and 23(b).

dark band lying between the two rainbows. To be complete in calculating the relative intensities of the two rainbows and the relative intensities of the colors in each rainbow, the other terms in $(23)$ besides $f^{2}(z)$ must be included. Also, the intensity distribution of the visible spectrum of sunlight or whatever artificial light source is used must be incorported. In addition, a Newtonian color chart would be required to determine the color shading that results from the overlap of the peaks for particular wavelengths. The peaks in the patterns would have to be widened because the sun, which subtends $0.5^{\circ}$ in our sky, does not give perfectly parallel incident rays. Finally, the variation in $I_{0}$ across the emerging wavefront, including its dependence on both the wavelength and the order of the rainbow, should be calculated.

Occasionally the interference pattern can be distinguished just below the natural primary rainbow and just above the natural secondary rainbow. Those infrequent additional bows are termed supernumerary bows, erroneously implying by the title that they should not be pres-

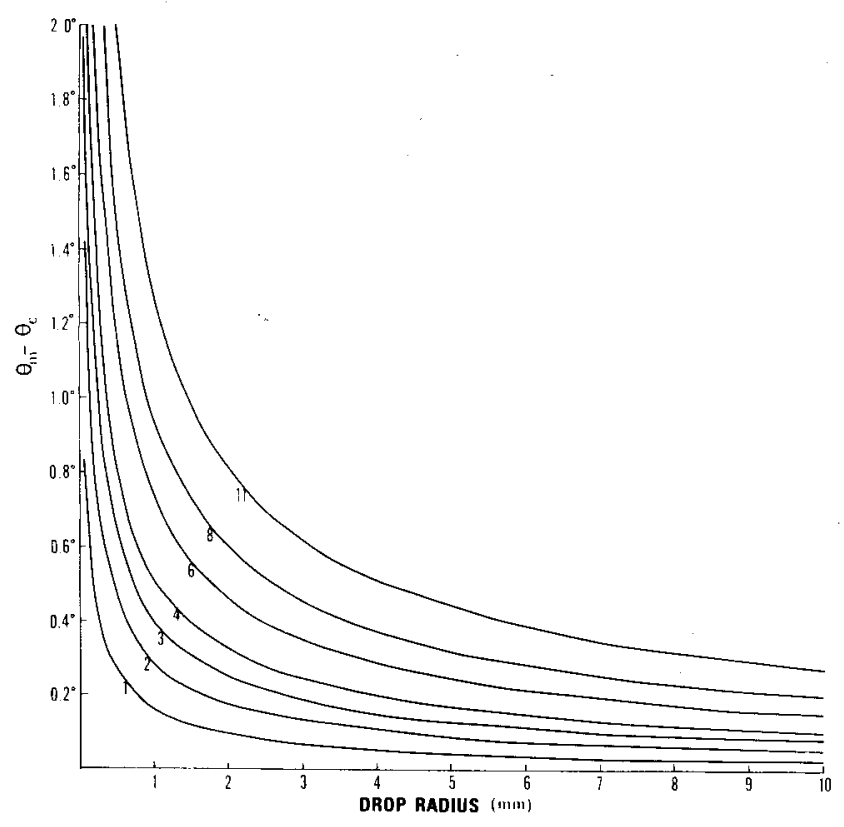

Fig. 25. The angular difference between the Cartesian angle $\theta_{c}$ and the principal maximum of the interference pattern $\theta_{m}$ is given for water drops of radii from 0.1 to $10 \mathrm{~mm}$ and for several of the first 11 rainbows.

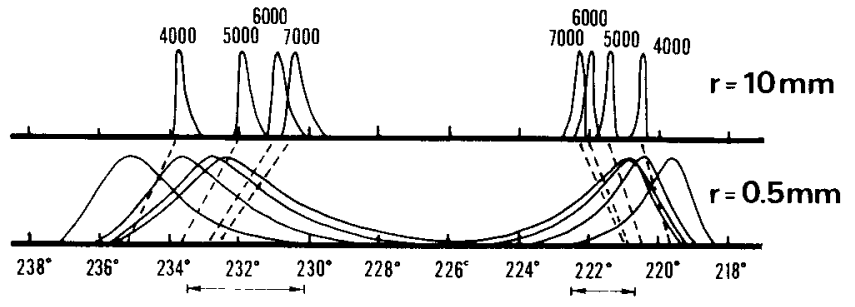

Fig. 26. Change in the rainbows with change in radius of the water drop. The color and wavelength sequence is the same as in Fig. 23(b). The Cartesian rainbows are indicated by the horizontal arrows.

ent. As a matter of fact, they belong to the same interference pattern as do the principal maxima.

Figure 24 is a photograph of the interference pattern from a suspended water drop, approximately $2 \mathrm{~mm}$ in diameter, when fully illuminated by a $\mathrm{He}-\mathrm{Ne}$ laser at $6328 \AA$. Both the primary and secondary patterns are seen, along with the intervening dark band. Similar patterns are visible for several of the higher-order rainbows from the drop. For the primary rainbow over 100 of the interference maxima can clearly be seen and measured for position. If the monochromatic laser light is replaced with either sunlight or artificial white light, the patterns become full-color rainbows, and the smaller interference maxima, the supernumeraries, are no longer so visible.

As shown in Fig. 23(a), the principal maxima do not occur exactly at the Cartesian rainbow angles. The difference between $\theta_{c}$ and the angle of the principal maximum $\theta_{m}$ depends on the size of the drop. With $z=1.0845$ to give the principal maximum, ${ }^{28}$ (24) and (26) become

$$
\theta_{m}-\theta_{c}=(1.0845 / \cos i)\left(\lambda^{2} \sin i / 64 a^{2}\right)^{1 / 3},
$$

which is graphed in Fig. 25 for drops with radii ranging from 0.1 to $10 \mathrm{~mm}$. The discrepancy between the waveinterference rainbows and the geometrical rainbows is significant for drops as large as $10 \mathrm{~mm}$ in radius, especially for the higher-order rainbows. For smaller drops, the discrepancy is even greater. However, the calculations for both geometrical and wave-interference rainbows may not apply for $0.1 \mathrm{~mm}$ or less, for it is near $0.1 \mathrm{~mm}$ that the Mie scattering theory is first required. ${ }^{11}$

The radius dependence of (27) and Fig. 25 can be obtained experimentally if a drop is illuminated with a single color. For example, at the $\mathrm{He}-\mathrm{Ne} 6328-\AA$ wavelength, the $\theta_{m}$ of the secondary rainbow typically increases by about $0.5^{\circ}$ with a decrease in radius of roughly $0.5 \mathrm{~mm}$, which takes about half an hour in the experimental setup described here. Corresponding changes are also seen in the rest of the interference pattern. The angular positions of the first, fifth, and tenth minima increase by $0.7^{\circ}, 1.1^{\circ}$, and $1.8^{\circ}$, respectively, for the same change in radius.

Not only do the peaks of the principal maxima shift away from $\theta_{c}$ as the drop radius is decreased, but the maxima also widen and hence overlap more (Fig. 26). This increased overlap eventually destroys the distinguishability of the colors. What one sees in the natural landscape, then, are white rainbows, single or double. With the suspended drops used in this work, the colors become noticeably duller and washed out as the drops evaporate and reach a radius of about $0.3 \mathrm{~mm}$. 


\section{ACKNOWLEDGMENTS}

I am very grateful to Professor K. J. Casper for several extensive reviews of this paper. The following students contributed to the initial development of the experiment: J. Baluk, E. Janson, and M. Strauss. The work was supported in part by an Instructional Assistance Grant from the Center for Effective Learning at Cleveland State University.

'From "Over the Rainbow," 1967, Leo Feist, Inc.

${ }^{2}$ For a bibliography of work on rainbows published through about 1958 , see C. B. Boyer, The Rainbow from Myth to Mathematics (Yoseloff, New York, 1959).

${ }^{3}$ For work published since 1958, see J. Walker, The Flying Circus of Physics (Wiley, New York, 1975), problems 5.32-5.41, 5.44.

${ }^{4} \mathrm{M}$. Minnaert, The Nature of Light and Colour in the Open Air (Dover, New York, 1954), pp. 174-176.

${ }^{3}$ See Ref. 2, pp. 209-217.

${ }^{6} \mathrm{~W}$. J. Humphreys, Physics of the Air (Dover, New York, 1964), pp. 476-480.

${ }^{7}$ H. C. Van de Hulst, Light Scattering by Small Particles (Wiley, New York, 1957), pp. 228-231, 240.

${ }^{8}$ R. A. R. Tricker, Introduction to Meteorological Optics (American Elsevier, New York, 1970), pp. 42-58.

${ }^{9} \mathrm{H}$. Neuberger, Introduction to Physical Meteorology (Pennsylvania State University, University Park, PA, 1957), pp. 174-179.
${ }^{10}$ R. J. Whitaker, Phys. Teach. 12, 283 (1974).

${ }^{11}$ K. Liou and J. E. Hansen, J. Atmos. Sci. 28, 995 (1971).

${ }^{12}$ J. Harsch and J. D. Walker, Am. J. Phys. 43, 453 (1975).

${ }^{13}$ See Ref. 2, p. 309 and Fig. 66.

${ }^{14}$ See Ref. 7, p. 240.

${ }^{15}$ See Ref. 2, p. 217.

${ }^{16}$ See Ref. 2, p. 241 ff.

${ }^{17}$ The natural rainbow is wider by about $0.5^{\circ}$ because the sun subtends about $0.5^{\circ}$ in our sky and thus does not give perfectly parallel incident rays of light.

${ }^{18}$ See Ref. 4, p. 179; Ref. 6, p. 482; Ref. 8, p. 56; Ref. 2, p. 215; Ref. 3, problem 5.35; and Ref. 12.

${ }^{19}$ See the plates in Ref. 2 and Plate 24 in D. H. McIntosh, Meteorological Glossary (Her Majesty's Stationery Office, London, 1972).

${ }^{20}$ See Ref. 7, pp. 203-205, and Ref. 8, pp. 64-68.

${ }^{2}$ S. Rösch, Appl. Opt. 7, 233 (1968).

${ }^{22}$ See Ref. 4, p. 181, and F. S. Crawford, Jr., Waves: Berkeley Physics Course, Vol. 3 (McGraw-Hill, New York, 1968), p. 419.

${ }^{23}$ The use of a spectrometer to observe the first and second rainbows from a drop suspended from the end of a wire is described by Tricker in Ref. 8 , p. 58.

${ }^{24}$ This liquid can be obtained from Fisher Scientific Co.

${ }^{25}$ See Ref. 6, pp. 488-495; Ref. 7, pp. 243-245; and Ref. 8, pp. 169 186.

${ }^{26}$ There is a reversal of the $u$ axis from that given in Ref. 6, p. 488; Ref. 7, p. 243; and Ref. 8, p. 177.

${ }^{27}$ See Ref. 6, p. 491 , and Ref. 8, p. 178.

${ }^{28}$ See Ref. 6, p. 492 . Abbreviated versions are in Ref. 7 , p. 244 , and Ref. 8, p. 179. 